\title{
Metabolism-related enzyme alterations identified by proteomic analysis in human renal cell carcinoma
}

\author{
This article was published in the following Dove Press journal: \\ OncoTargets and Therapy \\ 9 March 2016 \\ Number of times this article has been viewed
}

\author{
Zejun Lu',* \\ Yuqin Yao ${ }^{2, *}$ \\ Qi Song ${ }^{3}$ \\ Jinliang Yang ${ }^{4}$ \\ Xiangfei Zhao' \\ Ping Yang' \\ Jingbo Kang'
}

'Department of Radiation Oncology,

Naval General Hospital of People's

Liberation Army, Beijing, ${ }^{2}$ Research

Center for Public Health and

Preventive Medicine, West China

School of Public Health/No 4 West

China Teaching Hospital, Sichuan

University, Chengdu, ${ }^{3}$ Department

of Gynaecology and Obstetrics,

The General Hospital of Chinese

People's Armed Police Force, Beijing,

${ }^{4}$ State Key Laboratory of Biotherapy/

Collaborative Innovation Center

for Biotherapy, West China Hospital,

Sichuan University, Chengdu, People's

Republic of China

*These authors contributed equally to this work

Correspondence: Jingbo Kang

Department of Radiation Oncology,

Naval General Hospital of PLA,

Fuchengmen Road, No. 6, Beijing 100048 ,

Email kjbnet@I26.com
People's Republic of China

\begin{abstract}
The renal cell carcinoma (RCC) is one of the most common types of kidney neoplasia in Western countries; it is relatively resistant to conventional chemotherapy and radiotherapy. Metabolic disorders have a profound effect on the degree of malignancy and treatment resistance of the tumor. However, the molecular characteristics related to impaired metabolism leading to the initiation of RCC are still not very clear. In this study, two-dimensional electrophoresis (2-DE) and mass spectra (MS) technologies were utilized to identify the proteins involved in energy metabolism of RCC. A total of 73 proteins that were differentially expressed in conventional RCC, in comparison with the corresponding normal kidney tissues, were identified. Bioinformatics analysis has shown that these proteins are involved in glycolysis, urea cycle, and the metabolic pathways of pyruvate, propanoate, and arginine/proline. In addition, some were also involved in the signaling network of p53 and FAS. These results provide some clues for new therapeutic targets and treatment strategies of RCC.
\end{abstract}

Keywords: renal cell cancer, metabolism, two-dimensional electrophoresis, proteome

\section{Introduction}

The early stage diagnosis of renal cell carcinoma (RCC) in many countries is probably associated with the observed plateau in RCC mortality in the US and in many European countries. Nevertheless, $\sim 50 \%$ of patients diagnosed across all stages of this disease die within the first 5 years after diagnosis. ${ }^{1,2}$ Conventional chemotherapy and radiotherapy does not exert a significant long-term benefit on RCC; instead, it has been found to decrease the length or quality of life. RCC is not a single disease; rather, it is a compilation of several types of cancer that occur in the kidney. The poor prognosis of RCC is largely due to the effects of different oncogenes, each having a different histology and response to therapy. ${ }^{3}$ Metabolic control analysis is useful in assessing the influence of metabolic pathways on the course and treatment of complex diseases. ${ }^{4,5}$ Since the metabolic environment influences the rate-controlling steps of enzymes in metabolic pathways, the management of complex disease phenotypes is largely dependent on the expression of the entire collection of genes involved than on any particular gene or enzyme. ${ }^{6,7}$ This means that the management of complex disease phenotypes relies on a collection of system-wide interconnected processes that involve glycolysis and respiration. Successful manipulations of metabolic networks can lead to restoration of order and adaptive behavior in disordered states that involve complex gene-environment interactions. ${ }^{8,9}$

Metabolic control analysis is especially important in kidney cancer management, because disorder and abnormal energy metabolism are characteristics of RCC. ${ }^{10}$ However, there are few specific studies that identify the tumor-related metabolic proteins in RCC. In the present study, a comprehensive bioinformatics approach was applied to tissue proteomic data to identify those metabolic steps and networks

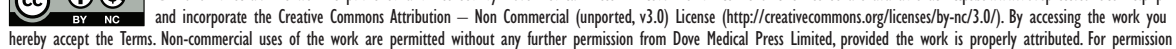
for commercial use of this work, please see paragraphs 4.2 and 5 of our Terms (https://www.dovepress.com/terms.php). 
that have a role in RCC onset and development. In kidney cancer, the expression of proteins involved in metabolism, cell growth, morphology, and the heat shock response is deregulated. Therefore, we hypothesize that the defects in identified pathways should serve as targets for the development of effective and long-lasting kidney cancer therapies that will be superior to those presently in use.

\section{Materials and methods}

\section{Tissue samples}

Surgical specimens of five patients from the Navy General Hospital, obtained after radical nephrectomy, were used to prepare tissue samples of conventional RCC and the surrounding noncancerous kidney tissues. The mean age of the patients was 55.8 years. The tumor stage of the patients ranged from $\mathrm{pT} 1$ to $\mathrm{pT} 3$. Macroscopic cell type of samples (benign or cancer) was examined histologically. The tumor stage was determined according to the 1997 TNM (tumor, node, metastasis) criteria. The samples were not necrotic. Table 1 shows a summary of detailed clinicopathologic data of patients included in the study. Institutional Ethics Committee of the Navy General Hospital approved this project, and informed consent were obtained from all patients, or their relatives, prior to commencing the study. A pathologist examined all specimens. The samples were immediately frozen in liquid nitrogen and stored at $-80^{\circ} \mathrm{C}$ until use.

\section{2-DE and image analysis}

Two-dimensional electrophoresis (2-DE) was performed as described previously. ${ }^{11}$ Briefly, cells were lysed in the lysis buffer (8 M urea, $2 \mathrm{M}$ thiourea, 4\% CHAPS, $100 \mathrm{mM}$ DTT, and $0.2 \%$ pH 3-10 ampholyte; Bio-Rad Laboratories Inc., Hercules, CA, USA) containing a protease inhibitor. After sonication and centrifugation, the supernatant was retrieved, and protein concentrations were determined using the DC protein assay kit (Bio-Rad Laboratories Inc.). Protein samples (1 mg) were applied to a immobilized $\mathrm{pH}$ gradient strip (17 cm, pH 3-10 non-linear [NL], Bio-Rad Laboratories Inc.) using a passive rehydration method. For the second dimension, a $30 \mathrm{~mA}$ constant current was applied to $12 \%$ sodium dodecyl

Table I Clinicopathologic features of renal cell carcinoma samples

\begin{tabular}{lllll}
\hline No & Sex & $\begin{array}{l}\text { Age } \\
\text { (years) }\end{array}$ & Clinicopathologic features & $\begin{array}{l}\text { TNM } \\
\text { stage }\end{array}$ \\
\hline I & Male & 52 & Clear cell renal cell carcinoma & TINOM0 \\
2 & Male & 52 & Clear cell renal cell carcinoma & TINOM0 \\
3 & Male & 60 & Clear cell renal cell carcinoma & T3NOMI \\
4 & Female & 54 & Clear cell renal cell carcinoma & T2NOM0 \\
5 & Male & 61 & Clear cell renal cell carcinoma & T2NOM0 \\
\hline
\end{tabular}

Abbreviation: TNM, tumor, node, metastasis. sulfate polyacrylamide gel electrophoresis gel after isoelectric focusing and equilibration. The gels were stained using $\mathrm{CBB}$ R-250 (EMD Millipore, Billerica, MA, USA) and scanned with a Bio-Rad GS-800 scanner (Bio-Rad Laboratories Inc.). The 2-DE analyses were independently repeated three times. The maps were analyzed by PDQuest software, Version 6.1 (Bio-Rad Laboratories Inc.). The quantity of each spot in the gel was normalized as the percentage of the total quantity of all spots in that gel and evaluated in terms of optical density (OD). The paired $t$-test was performed to compare the data from three repeated experiments. Only those spots that showed consistent and significant differences $(>1.5$-fold, $P<0.05)$ were selected for further analysis with mass spectra (MS).

\section{In-gel digestion}

In-gel digestion of proteins was performed using mass spectrometry grade Trypsin Gold (Promega Corporation, Madison, WI, USA). Briefly, the spots were cut out of the gel (1-2 mm diameter) using a razor blade and destained twice with $100 \mathrm{mM} \mathrm{NH}_{4} \mathrm{HCO}_{3} / 50 \%$ acetonitrile $(\mathrm{ACN})$ at $37^{\circ} \mathrm{C}$ for 45 minutes in each treatment. After drying, the gels were preincubated in 10-20 $\mu \mathrm{L}$ trypsin solution for 1 hour. Following, $15 \mu \mathrm{L}$ digestion buffer was added (40 mM NH $\mathrm{HCO}_{3} / 10 \%$ ACN) to cover each gel and incubated overnight at $37^{\circ} \mathrm{C}$. Tryptic digests were extracted using Milli-Q water initially, followed by two 1 hour repeat extractions with 50\% ACN/5\% trifluoroacetic acid. The combined extracts were dried in a vacuum concentrator at room temperature. The samples were then subjected to mass spectrometric analysis.

\section{MS/MS analysis and protein identification}

Mass spectra were acquired using a quadrupole time-of-flight mass spectrometer (Micromass, Manchester, UK) fitted with an electrospray ionization or matrix-assisted laser desorption/ionization source (Micromass). The MS/MS analysis was performed as described previously. ${ }^{12}$ The MS/MS data were acquired and processed using MassLynx V 4.1 software (Micromass) and converted to PKL performed using ProteinLynx 2.2.5 software (Waters Corp, Milford, MA, USA). The pkl files were analyzed using the MASCOT search engine (http://www.matrixscience.com). The following search parameters were used: database, Swiss-Prot, taxonomy, Homo sapiens, enzyme, and trypsin. One missed cleavage was allowed. Carbamidomethylation was selected as a fixed modification, and oxidation of methionine was set as the variable. The peptide and fragment mass tolerance were set at $0.1 \mathrm{Da}$ and $0.05 \mathrm{Da}$, respectively. Positively identified proteins had at least one peptide exceeding their 
score threshold $(P<0.05)$, and their molecular weight and isoelectric point consistent with the gel regions from which the spots were excised. The spectra of proteins identified by a single peptide, and with a score $>40$ (lower were discarded) were manually inspected.

\section{Immunoblot}

The radioimmunoprecipitation assay lysis buffer $(50 \mathrm{mM}$ Tris- $\mathrm{HCl}$ [pH 7.4], 1\% NP-40, 0.25\% Na-deoxycholate, $150 \mathrm{mM} \mathrm{NaCl}, 1 \mathrm{mM}$ ethylenediaminetetraacetic acid, $1 \mathrm{mM}$ phenylmethylsulfonyl fluoride, $1 \mathrm{mg} / \mathrm{mL}$ aprotinin, $1 \mathrm{mM}$ $\mathrm{Na}_{3} \mathrm{VO}_{4}$, and $1 \mathrm{mM} \mathrm{NaF}$ ) was used to break open the cells. The proteins were then suspended in the Lammli sample buffer and centrifuged at 15,000 rpm for 30 minutes. The supernatant was recovered for analysis. Each protein sample of $10 \mu \mathrm{g}$ was loaded per well and separated with $12.5 \%$ sodium dodecyl sulfate polyacrylamide gel electrophoresis. The proteins inside the gel were electroblotted onto polyvinylidene fluoride membranes (EMD Millipore) by wet blotting. After incubation in the blocking buffer $(1 \times$ Tris-buffered saline, $0.1 \%$ Tween- 20 , and $5 \% \mathrm{w} / \mathrm{v}$ dry nonfat milk) for 1 hour at room temperature, the membranes were incubated by primary antibodies. Following, the membrane were incubated with secondary antibodies for 45 minutes at room temperature. Enhanced chemiluminescence was used to detect reactive bands (Amersham Biosciences Corp, Piscataway, NJ, USA).

\section{Bioinformatics and statistical analysis}

Gene Ontology search was used to (www.geneontology.org) classify and determine the functions of identified proteins. Pathway data were obtained from the Kyoto Encyclopedia of Genes and Genomes (http://www.genome.jp/kegg) a collection of online databases dealing with genomes, enzymatic pathways, and biological chemicals. ${ }^{13}$ Protein-protein interactions were identified with the search tool STRING database. Both direct (physical) and indirect (functional) protein associations were examined. ${ }^{14,15}$

The two-tailed Student's $t$-test was used to determine the significant differences between the control and the exposure groups. Statistical analysis was performed using SPSS 16.0 software (SPSS Inc., Chicago, IL, USA), and $P<0.05$ was considered statistically significant.

\section{Results}

\section{2-DE profiling of differentially expressed proteins}

Protein expression in RCC tissues and the corresponding normal kidney tissues was examined by 2-DE. Figure 2 shows a pair of representative 2-DE maps. Proteins extracted from RCC tissues and the corresponding normal kidney tissues was resolved by 2-DE and visualized by CBB R-250 staining. Those protein spots with a $P$-value $<0.05$ according to the Student's $t$-test and reproducible changes in intensity $>1.5$ fold were identified. The analysis resulted in a total of 90 protein spots that were differentially expressed between RCC tissues and the corresponding normal kidney tissues; of those, 73 proteins were successfully identified by MS/MS (Table 2). Twenty-six proteins were downregulated and 47 proteins were upregulated in RCC tissues (Figures 1 and 2).

\section{Protein identification and functional classification}

Seventy-three proteins were identified (Figure 1) and are listed Table 2. The MS/MS data, which included the mass and intensity values, and the charge of the precursor ions, were compared against the SWISS-PROT protein database using a licensed copy of the MASCOT 2.0 program. Figure 3 shows a representative MS/MS map of spot \#9. Among them, $\mathrm{HIBCH}$ was downregulated in RCC tissues in comparison with the adjacent nonmalignant tissues $(P<0.05)$. Furthermore, MS/MS analysis revealed 12 matching peptides, with $44 \%$ sequence coverage and a MOWSE score of 714 (Figure 3).

\section{Immunoblotting validation for differentially expressed proteins}

Two altered proteins, EIF5A and PKM2, were further validated by Western blotting. As shown in Figure 4, EIF5A and PKM2 were upregulated in RCC tissue in comparison with adjacent nonmalignant tissue, which was consistent with the 2-DE results $(P<0.05)$.

\section{Network, pathway, and process analyses of significantly changed proteins}

Table 2 lists differentially expressed proteins in RCC and the corresponding normal tissues, as confirmed by mass spectrometry. Their molecular function and biological processes are included in Table 2. Interactions exist among these proteins, and most of them are a part of a biological network, as illustrated by STRING (Figure 5). Out of 73 identified proteins, 63 were interconnected and ten proteins did not show any type of connection at the selected confidence level (STRING score =0.4). The following significant functions are associated with this network of proteins: metabolism, transcription, proteolysis, electron transport, and molecular chaperoning. ENO1, ENO2, AKR1A1, PGAM1, and PGA are important proteins in gluconeogenesis, while 
Table 2 Identification results of proteins differentially expressed between RCC and the corresponding normal tissues

\begin{tabular}{|c|c|c|c|c|c|c|c|c|}
\hline $\begin{array}{l}\text { Spot } \\
\text { no }\end{array}$ & Protein description & $\begin{array}{l}\text { Gene } \\
\text { name }^{a}\end{array}$ & Function & $\begin{array}{l}\text { Accession } \\
\text { no }^{\text {b }}\end{array}$ & $\begin{array}{l}\text { Theoreticalc } \\
\text { MW/pl }\end{array}$ & Score $^{d}$ & Coverage $^{e}$ & $\begin{array}{l}\text { Fold } \\
\text { change }{ }^{f}\end{array}$ \\
\hline I & Phosphoenolpyruvate carboxykinase [GTP] & PCK2 & Metabolism & Q16822 & $7 \mid, 438 / 7.57$ & 233 & $22 \%$ & $\downarrow$ \\
\hline 2 & $78 \mathrm{kDa}$ glucose-regulated protein & GRP78 & $\begin{array}{l}\text { Molecular } \\
\text { chaperone }\end{array}$ & PII02I & $72,402 / 5.07$ & 58 & $6 \%$ & $\downarrow$ \\
\hline 3 & $\begin{array}{l}\text { Delta-I-pyrroline-5-carboxylate } \\
\text { dehydrogenase }\end{array}$ & ALDH4AI & Metabolism & P30038 & $62,137 / 8.25$ & 224 & $21 \%$ & $\downarrow$ \\
\hline 4 & $\begin{array}{l}\text { Alpha-aminoadipic semialdehyde } \\
\text { dehydrogenase }\end{array}$ & ALDH7AI & Metabolism & P49419 & $59,020 / 8.21$ & 255 & $22 \%$ & $\downarrow$ \\
\hline 5 & Glycine amidinotransferase & GATM & Metabolism & P50440 & $48,938 / 8.26$ & 167 & $42 \%$ & $\downarrow$ \\
\hline 6 & $\begin{array}{l}\text { Medium-chain specific acyl-CoA } \\
\text { dehydrogenase }\end{array}$ & ACADM & Metabolism & $\mathrm{PII} 1310$ & $47,015 / 8.61$ & 302 & $26 \%$ & $\downarrow$ \\
\hline 7 & Argininosuccinate synthase & ASS & Metabolism & P00966 & $46,786 / 8.08$ & 230 & $33 \%$ & $\downarrow$ \\
\hline 8 & Fructose-1,6-bisphosphatase I & FBPI & Metabolism & P09467 & $37,218 / 6.54$ & I,094 & $56 \%$ & $\downarrow$ \\
\hline 9 & 3-Hydroxyisobutyryl-CoA hydrolase & $\mathrm{HIBCH}$ & Metabolism & Q6NVYI & $43,797 / 8.38$ & 714 & $44 \%$ & $\downarrow$ \\
\hline 10 & $\begin{array}{l}\text { Acetyl-CoA acetyltransferase, } \\
\text { mitochondrial }\end{array}$ & ACATI & Metabolism & P24752 & $45,456 / 8.98$ & 585 & $39 \%$ & $\downarrow$ \\
\hline 11 & Ester hydrolase $\mathrm{CI}$ lorf54 & $\mathrm{Cl}$ lorf54 & Metabolism & Q9HOW9 & $35,608 / 6.23$ & 240 & $31 \%$ & $\downarrow$ \\
\hline 12 & $\begin{array}{l}\text { Glycerol-3-phosphate dehydrogenase } \\
{[N A D+]}\end{array}$ & GPDI & Metabolism & P21695 & $38,|7| / 5.81$ & 863 & $60 \%$ & $\downarrow$ \\
\hline 13 & $\begin{array}{l}\text { Complement component I Q } \\
\text { subcomponent-binding protein }\end{array}$ & $C I Q B P$ & $\begin{array}{l}\text { Immune } \\
\text { regulation }\end{array}$ & P0702I & $31,749 / 4.74$ & 2,109 & $46 \%$ & $\downarrow$ \\
\hline 14 & Agmatinase, mitochondrial & AGMAT & Metabolism & Q9BSE5 & $38,206 / 7.55$ & 707 & $41 \%$ & $\downarrow$ \\
\hline 15 & Calbindin & CALBI & Metabolism & P05937 & $30,291 / 4.7$ & 531 & $46 \%$ & $\downarrow$ \\
\hline 16 & Glutathione peroxidase 3 & GPX3 & Metabolism & P22352 & $25,765 / 8.26$ & 491 & $29 \%$ & $\downarrow$ \\
\hline 17 & Cytochrome b-cl complex subunit Rieske & UQCRFSI & $\begin{array}{l}\text { Electron } \\
\text { transport }\end{array}$ & P47985 & $29,934 / 8.55$ & 405 & $37 \%$ & $\downarrow$ \\
\hline 18 & ESI protein homolog & C2 I orf33 & Metabolism & P30042 & $28,495 / 8.5$ & 201 & $38 \%$ & $\downarrow$ \\
\hline 19 & Transgelin & TAGLN & $\begin{array}{l}\text { Structural } \\
\text { component }\end{array}$ & P01995 & $22,653 / 8.87$ & 158 & $57 \%$ & $\downarrow$ \\
\hline 20 & Nucleoside diphosphate kinase B & NME2 & Metabolism & P22392 & $|7,40| / 8.52$ & 205 & $40 \%$ & $\downarrow$ \\
\hline 21 & Nucleoside diphosphate kinase A & NMEI & Metabolism & PI553I & $17,309 / 5.83$ & 246 & $53 \%$ & $\downarrow$ \\
\hline 22 & Peptidyl-prolyl cis-trans isomerase B & PPIB & Metabolism & P23284 & $23,785 / 9.42$ & 511 & $41 \%$ & $\downarrow$ \\
\hline 23 & Transthyretin & TTR & $\begin{array}{l}\text { Hormone-binding } \\
\text { protein }\end{array}$ & P02766 & $|5,99| / 5.52$ & 122 & $40 \%$ & $\downarrow$ \\
\hline 24 & $\begin{array}{l}\text { Cytochrome c oxidase subunit } 5 \mathrm{~A} \text {, } \\
\text { mitochondrial }\end{array}$ & $\operatorname{coX} 5 A$ & $\begin{array}{l}\text { Electron } \\
\text { transport }\end{array}$ & P20674 & $16,923 / 6.3$ & 435 & $47 \%$ & $\downarrow$ \\
\hline 25 & Fatty acid-binding protein, liver & FABPI & Lipid transport & P07।48 & $14,256 / 6.6$ & 368 & $53 \%$ & $\downarrow$ \\
\hline 26 & $10 \mathrm{kDa}$ heat shock protein & HSPEI & Metabolism & $\mathrm{P} 61604$ & $10,925 / 8.89$ & 533 & $52 \%$ & $\downarrow$ \\
\hline 27 & Serum albumin & $A L B$ & Metabolism & P02768 & $71,317 / 5.92$ & 195 & $16 \%$ & $\uparrow$ \\
\hline 28 & Retinal dehydrogenase I & ALDHI & Metabolism & P00352 & $55,454 / 6.3$ & 1,196 & $59 \%$ & $\uparrow$ \\
\hline 29 & Alpha-enolase & ENOI & Metabolism & Q6GMP2 & $47,481 / 7.01$ & 2,799 & $65 \%$ & $\uparrow$ \\
\hline 30 & Glycine amidinotransferase, mitochondrial & GATM & Metabolism & P50440 & $48,938 / 8.26$ & 288 & $49 \%$ & $\uparrow$ \\
\hline 31 & Pyruvate kinase isozymes $\mathrm{MI} / \mathrm{M} 2$ & KPYM & Metabolism & Q9BWB5 & $58,480 / 7.96$ & 259 & $29 \%$ & $\uparrow$ \\
\hline 32 & Septin-2 & SEPT2 & $\begin{array}{l}\text { Structural } \\
\text { component }\end{array}$ & Q15019 & $41,689 / 6.15$ & 519 & $40 \%$ & $\uparrow$ \\
\hline 33 & Fumarylacetoacetase & $\mathrm{FAH}$ & Metabolism & $\mathrm{P} 16930$ & $46,743 / 6.46$ & 425 & $30 \%$ & $\uparrow$ \\
\hline 34 & Gamma-enolase & ENO2 & Metabolism & P09104 & $47,581 / 4.91$ & 771 & $7 \%$ & $\uparrow$ \\
\hline 35 & Phosphotriesterase-related protein & PTER & Metabolism & Q96BW5 & $39,506 / 6.07$ & 201 & $59 \%$ & $\uparrow$ \\
\hline 36 & Alpha-soluble NSF attachment protein & NAPA & $\begin{array}{l}\text { Electron } \\
\text { transport }\end{array}$ & P54920 & $33,667 / 5.23$ & 228 & $66 \%$ & $\uparrow$ \\
\hline 37 & Annexin A4 & ANXA4 & $\begin{array}{l}\text { Calcium ion } \\
\text { binding }\end{array}$ & P09525 & $36,092 / 5.84$ & 985 & $54 \%$ & $\uparrow$ \\
\hline 38 & Phosphoserine aminotransferase & PSATI & Metabolism & Q9Y617 & $40,796 / 7.56$ & 249 & $44 \%$ & $\uparrow$ \\
\hline 39 & Aldose reductase & ALDRI & Metabolism & PI5I2I & $36,230 / 6.5 I$ & 293 & $68 \%$ & $\uparrow$ \\
\hline 40 & Annexin A2 & ANXA2 & $\begin{array}{l}\text { Calcium ion } \\
\text { binding }\end{array}$ & P07355 & $38,808 / 7.57$ & 359 & $46 \%$ & $\uparrow$ \\
\hline
\end{tabular}


Table 2 (Continued)

\begin{tabular}{|c|c|c|c|c|c|c|c|c|}
\hline $\begin{array}{l}\text { Spot } \\
\text { no }\end{array}$ & Protein description & $\begin{array}{l}\text { Gene } \\
\text { name }^{a}\end{array}$ & Function & $\begin{array}{l}\text { Accession } \\
\text { no }^{\mathrm{b}}\end{array}$ & $\begin{array}{l}\text { Theoreticalc } \\
\text { MW/pl }\end{array}$ & Score $^{d}$ & Coverage $^{\mathrm{e}}$ & $\begin{array}{l}\text { Fold } \\
\text { change }^{f}\end{array}$ \\
\hline $4 \mathrm{II}$ & Proteasome activator complex subunit 2 & PSME2 & Proteolysis & Q9UL46 & $27,555 / 5.54$ & 584 & $66 \%$ & $\uparrow$ \\
\hline 42 & Proteasome subunit alpha type- 3 & PSMA3 & Proteolysis & P25788 & $28,643 / 5.19$ & 224 & $14 \%$ & $\uparrow$ \\
\hline 43 & S-formylglutathione hydrolase & ESD & Metabolism & PI0768 & $31,956 / 6.54$ & 276 & $47 \%$ & $\uparrow$ \\
\hline 44 & $\begin{array}{l}\text { Voltage-dependent anion-selective channel } \\
\text { protein } 2\end{array}$ & VDAC2 & $\begin{array}{l}\text { Electron } \\
\text { transport }\end{array}$ & P45880 & $32,060 / 7.49$ & 235 & $40 \%$ & $\uparrow$ \\
\hline 45 & Nicotinamide $\mathrm{N}$-methyltransferase & NNMT & Metabolism & P4026I & $30,011 / 5.56$ & 648 & $50 \%$ & $\uparrow$ \\
\hline 46 & Glutathione $\mathrm{S}$-transferase $\mathrm{P}$ & GSTPI & Metabolism & P092II & $23,569 / 5.43$ & $\mathrm{I}, 197$ & $85 \%$ & $\uparrow$ \\
\hline 47 & Proteasome subunit beta type- 4 & PSMB4 & Proteolysis & P28070 & $29,242 / 5.72$ & 436 & $38 \%$ & $\uparrow$ \\
\hline 48 & Phosphoglycerate mutase I & PGAMI & Metabolism & PI8669 & $28,900 / 6.67$ & 197 & $45 \%$ & $\uparrow$ \\
\hline 49 & Triosephosphate isomerase & TPI & Metabolism & $\mathrm{P} 60174$ & $26,943 / 6.45$ & 1,109 & $55 \%$ & $\uparrow$ \\
\hline 50 & Superoxide dismutase [Mn] & SOD2 & $\begin{array}{l}\text { Electron } \\
\text { transport }\end{array}$ & P04I79 & $24,722 / 8.35$ & 442 & $59 \%$ & $\uparrow$ \\
\hline 51 & Proteasome subunit beta type- 8 & PSMB8 & Proteolysis & P28062 & $30,677 / 7.67$ & 273 & $27 \%$ & $\uparrow$ \\
\hline 52 & Actin, cytoplasmic I & ACTB & $\begin{array}{l}\text { Structural } \\
\text { component }\end{array}$ & P60709 & $42,052 / 5.29$ & 194 & $7 \%$ & $\uparrow$ \\
\hline 53 & Alpha-I-antitrypsin & AAT & Metabolism & P0I009 & $46,878 / 5.37$ & 44 & $3 \%$ & $\uparrow$ \\
\hline 54 & Sorcin & $S R I$ & $\begin{array}{l}\text { Calcium ion } \\
\text { binding }\end{array}$ & P30626 & $21,947 / 5.32$ & 237 & $47 \%$ & $\uparrow$ \\
\hline 55 & Ferritin heavy chain & FTHI & Metabolism & P02794 & $21,383 / 5.3$ & 110 & $22 \%$ & $\uparrow$ \\
\hline 56 & Haptoglobin & $H P$ & Metabolism & P00738 & $45,861 / 6.13$ & 139 & $14 \%$ & $\uparrow$ \\
\hline 57 & Alpha-crystallin B chain & $C R Y A B$ & Metabolism & P025II & $20,146 / 6.76$ & 772 & $72 \%$ & $\uparrow$ \\
\hline 58 & Hippocalcin-like protein I & HPCALI & Metabolism & P37235 & $22,4|3 / 5.2|$ & 108 & $29 \%$ & $\uparrow$ \\
\hline 59 & Ferritin light chain & FTL & Metabolism & P02792 & $20,064 / 5.51$ & 391 & $41 \%$ & $\uparrow$ \\
\hline 60 & Eukaryotic translation initiation factor $5 \mathrm{~A}-\mathrm{I}$ & EIF5A & $\begin{array}{l}\text { Translation } \\
\text { regulation }\end{array}$ & P6324I & $17,053 / 5.08$ & 303 & $42 \%$ & $\uparrow$ \\
\hline 61 & Matrilysin & MMP7 & Metabolism & P09237 & $29,829 / 7.74$ & 181 & $35 \%$ & $\uparrow$ \\
\hline 62 & Cofilin-I & COFI & $\begin{array}{l}\text { Signal } \\
\text { transduction }\end{array}$ & P23528 & $18,723 / 8.22$ & 364 & $31 \%$ & $\uparrow$ \\
\hline 63 & Peptidyl-prolyl cis-trans isomerase A & PPIA & Protein folding & P62937 & $18,233 / 7.68$ & 548 & $64 \%$ & $\uparrow$ \\
\hline 64 & Annexin $\mathrm{A} 3$ & ANXA3 & $\begin{array}{l}\text { Calcium ion } \\
\text { binding }\end{array}$ & PI2429 & $36,527 / 5.63$ & 87 & $4 \%$ & $\uparrow$ \\
\hline 65 & Fatty acid-binding protein, epidermal & FABP5 & $\begin{array}{l}\text { Structural } \\
\text { component }\end{array}$ & Q01469 & $15,497 / 6.6$ & 291 & $43 \%$ & $\uparrow$ \\
\hline 66 & Histidine triad nucleotide-binding protein I & HINTI & Metabolism & P49773 & $13,905 / 6.43$ & 477 & $65 \%$ & $\uparrow$ \\
\hline 67 & Glutathione S-transferase theta-I & GSTTI & Metabolism & P307II & $27,489 / 7.01$ & 125 & $13 \%$ & $\uparrow$ \\
\hline 68 & Small nuclear ribonucleoprotein $\mathrm{F}$ & SNRPF & Metabolism & P62306 & $97,76 / 4.7$ & 161 & $40 \%$ & $\uparrow$ \\
\hline 69 & $\begin{array}{l}\text { SH3 domain-binding glutamic acid-rich-like } \\
\text { protein } 3\end{array}$ & SH3BGRL3 & Metabolism & Q9H299 & $10,488 / 4.82$ & 194 & $66 \%$ & $\uparrow$ \\
\hline 70 & Protein SI00-A4 & SIOOA4 & $\begin{array}{l}\text { Calcium ion } \\
\text { binding }\end{array}$ & P26447 & $11,949 / 5.85$ & 367 & $49 \%$ & $\uparrow$ \\
\hline 71 & Protein SI00-AII & SIOOAII & $\begin{array}{l}\text { Calcium ion } \\
\text { binding }\end{array}$ & Р31949 & $11,847 / 6.56$ & $\mathrm{I}, 792$ & $73 \%$ & $\uparrow$ \\
\hline 72 & Beta-2-microglobulin & $B 2 M$ & $\begin{array}{l}\text { Immune } \\
\text { regulation }\end{array}$ & P61769 & $13,820 / 6.06$ & 402 & $37 \%$ & $\uparrow$ \\
\hline 73 & Ubiquitin-40S ribosomal protein S27a & RPS27A & Metabolism & P62979 & $18,296 / 9.68$ & 78 & $24 \%$ & $\uparrow$ \\
\hline
\end{tabular}

Notes: a,bthe proteins gene name and ID from ExPASy database; 'theoretical molecular weight (kDa) and pl from the ExPASy database; ${ }^{d}$ probability-based MOWSE scores; enumber of unique peptides identified by MS/MS sequencing and sequence coverage; 'expression level in RCC compared with the corresponding normal tissues. $\uparrow$, increase; $\downarrow$, decrease.

Abbreviations: MW, molecular weight; pl, isoelectric point; MS, mass spectra; RCC, renal cell carcinoma.

PSME2, PSMA3, PSMB4, and PSMB8 are involved in proteasome-related proteolysis. The proteins were divided into several classes as a result of bioinformatic analysis based on the Kyoto Encyclopedia of Genes and Genomes pathway, which included: gluconeogenesis, the urea cycle and amino acid metabolism, proteasome, fatty acid metabolism, glutathione (GSH) metabolism, and so forth (Table 3).

\section{Discussion}

Identifying the changes in protein expression in cancer cells is a useful predictor of potential changes in the functional 

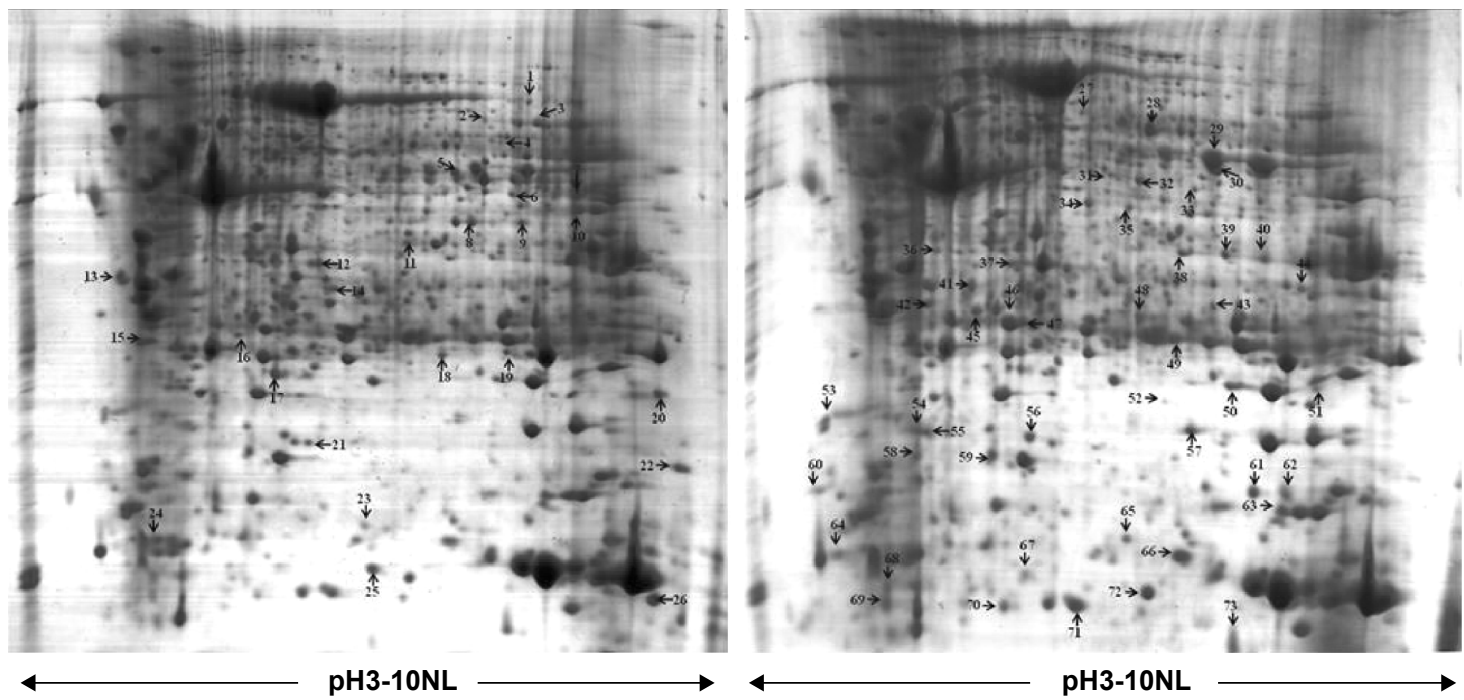

Figure I Representative 2-DE gel images of RCC tissue compared to adjacent nonmalignant tissue.

Notes: The gels were stained with Coomassie brilliant blue R250. Differentially expressed protein spots were labeled with numbers.

Abbreviations: RCC, renal cell carcinoma; 2-DE, two-dimensional electrophoresis.

pathways, which are, in turn, directly related to the basic mechanism of cancer onset and progression. Analysis at the proteome level enables the identification of proteins that are differentially expressed in RCC and adjacent normal tissues. These RCC-specific protein biomarkers might facilitate more efficient subclassification and early diagnosis of RCC. ${ }^{16,17}$ In this study, we analyzed the expression of proteins in eleven pairs of RCC tissues and matching normal kidney tissues from RCC patients utilizing two-dimensional electrophoresis and matrix-assisted laser
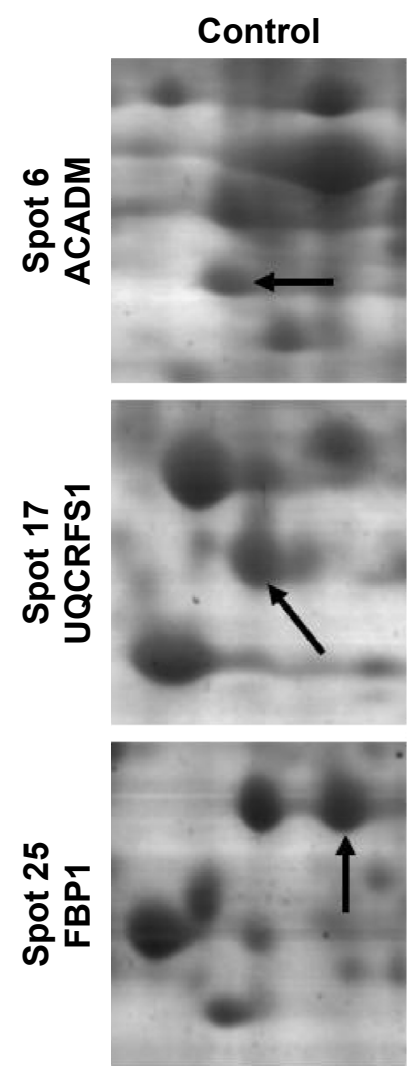

Cancer
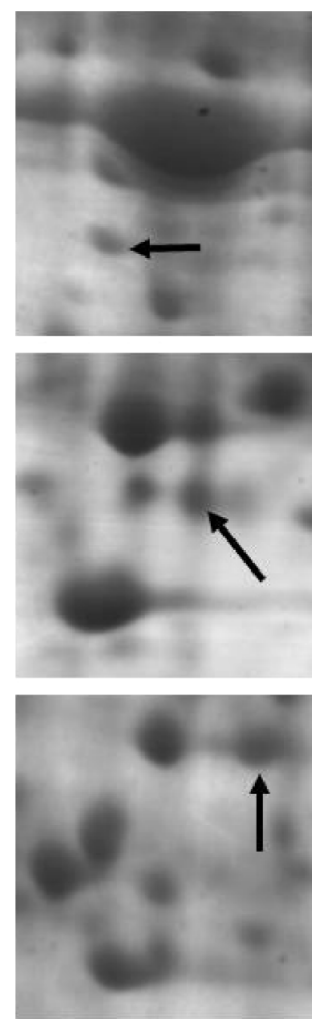
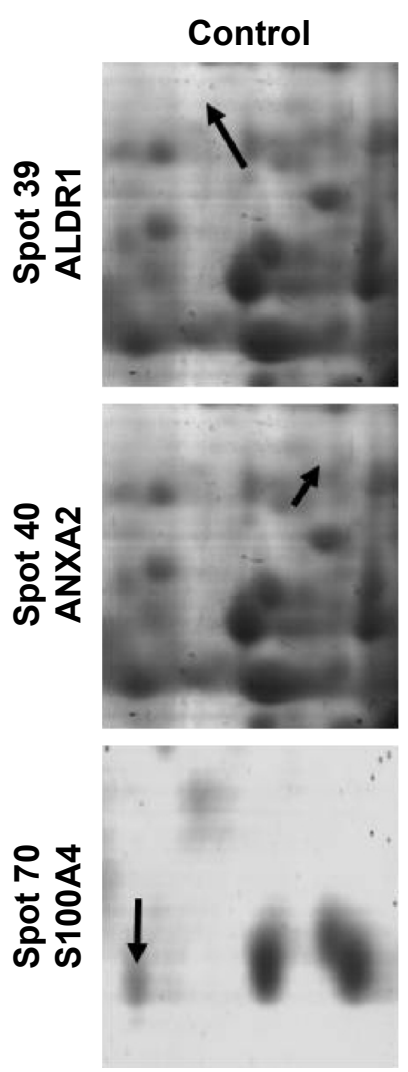

\section{Cancer}
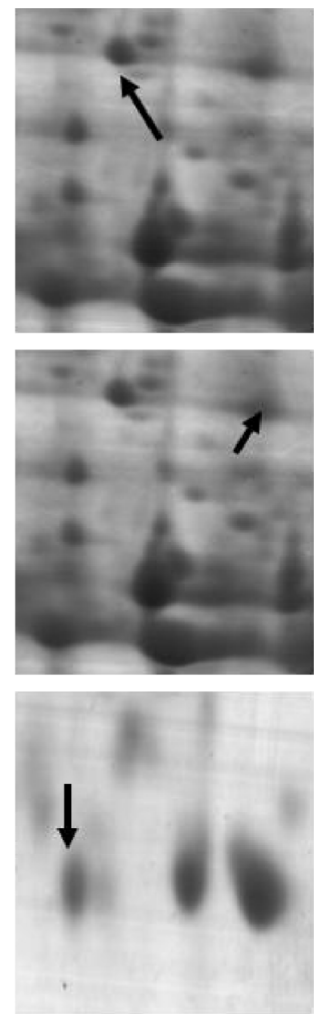

Figure 2 The enlargement of six selected regions as examples of protein spots that are dysregulated in this study. Notes: Protein spot discrepancies were labeled with arrows and marked with numbers (to the left of the images). 


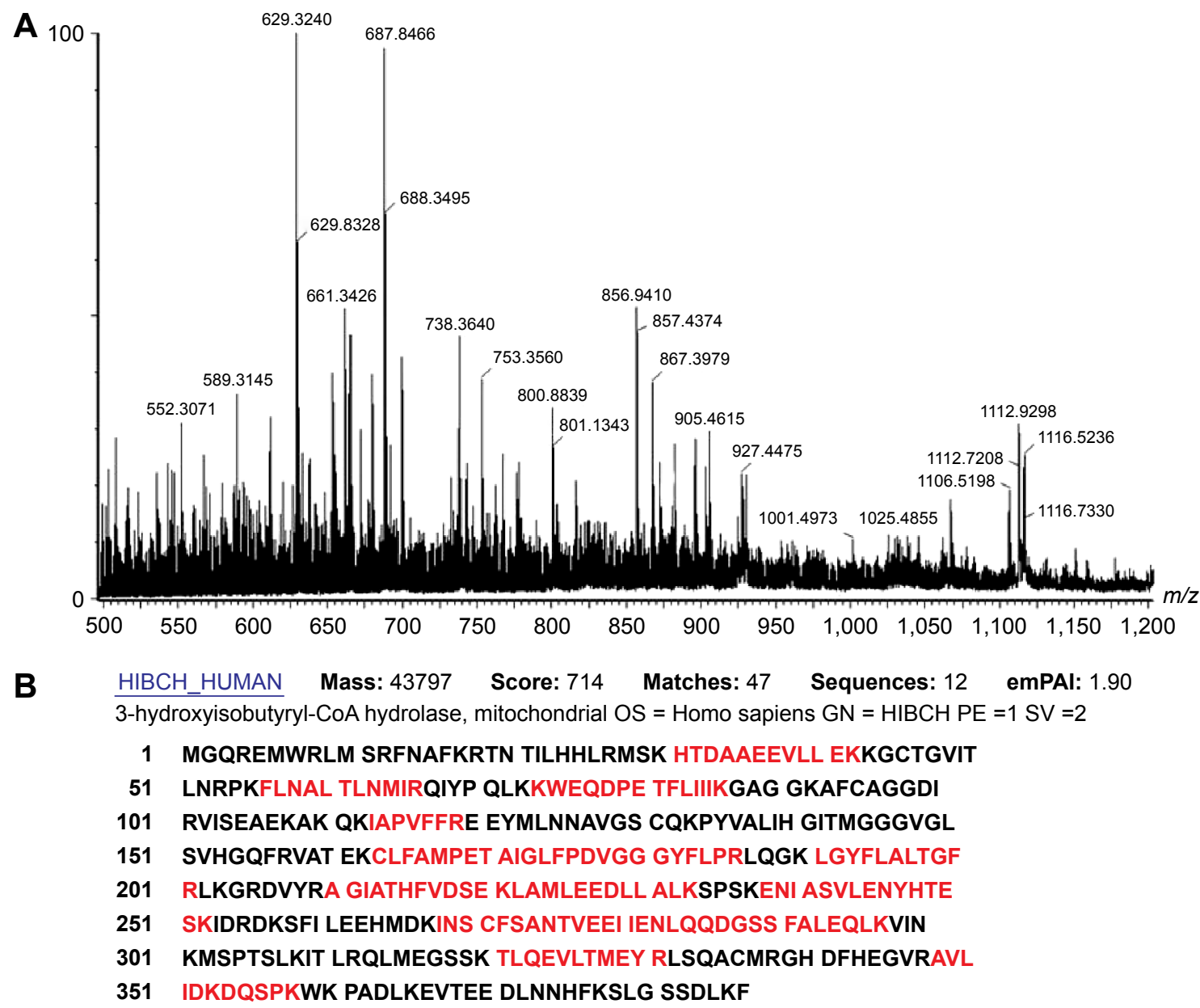

Figure 3 Identification of protein spot \#9.

Notes: (A) Peptide mass fingerprinting (PMF) of protein HIBCH. (B) HIBCH was identified by searching the MS/MS database using the MASCOT program. The matching peptides are shown in bold red.

Abbreviations: $\mathrm{HIBCH}, 3-\mathrm{Hydroxy}$ isobutyryl-CoA Hydrolase; MS, mass spectra.

desorption/ionization time-of-flight mass spectrometry. We found that 47 proteins were overexpressed and 26 proteins underexpressed in RCC. An altered expression of some of these proteins has previously been observed in RCC. ${ }^{18}$ The analysis of biochemical pathways conducted in this study has led to identification of protein networks, which play important roles in oncogenesis or progression of clear cell RCC (ccRCC).
A

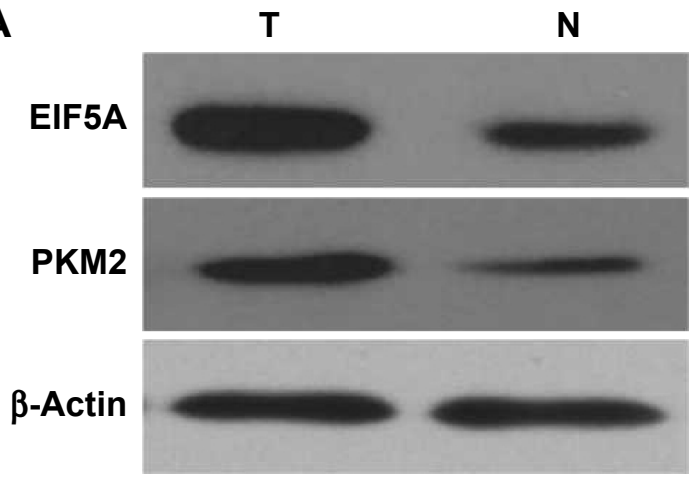

B

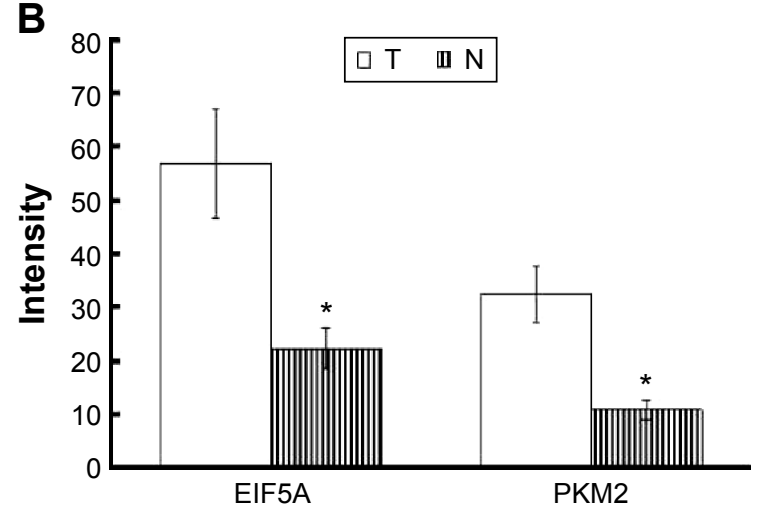

Figure 4 Western bolt detection of EIF5A and PKM2 expression in RCC tissue (T) and adjacent non-malignant tissue (N).

Notes: (A) EIF5A and PKM2 were upregulated in RCC tissue. (B) Western blot data were quantified densitometrically and $\beta$-actin was used as the loading control. Data are expressed as mean \pm SD from three independent experiments. $* P<0.05$, compared with adjacent non-malignant tissue.

Abbreviation: RCC, renal cell carcinoma. 


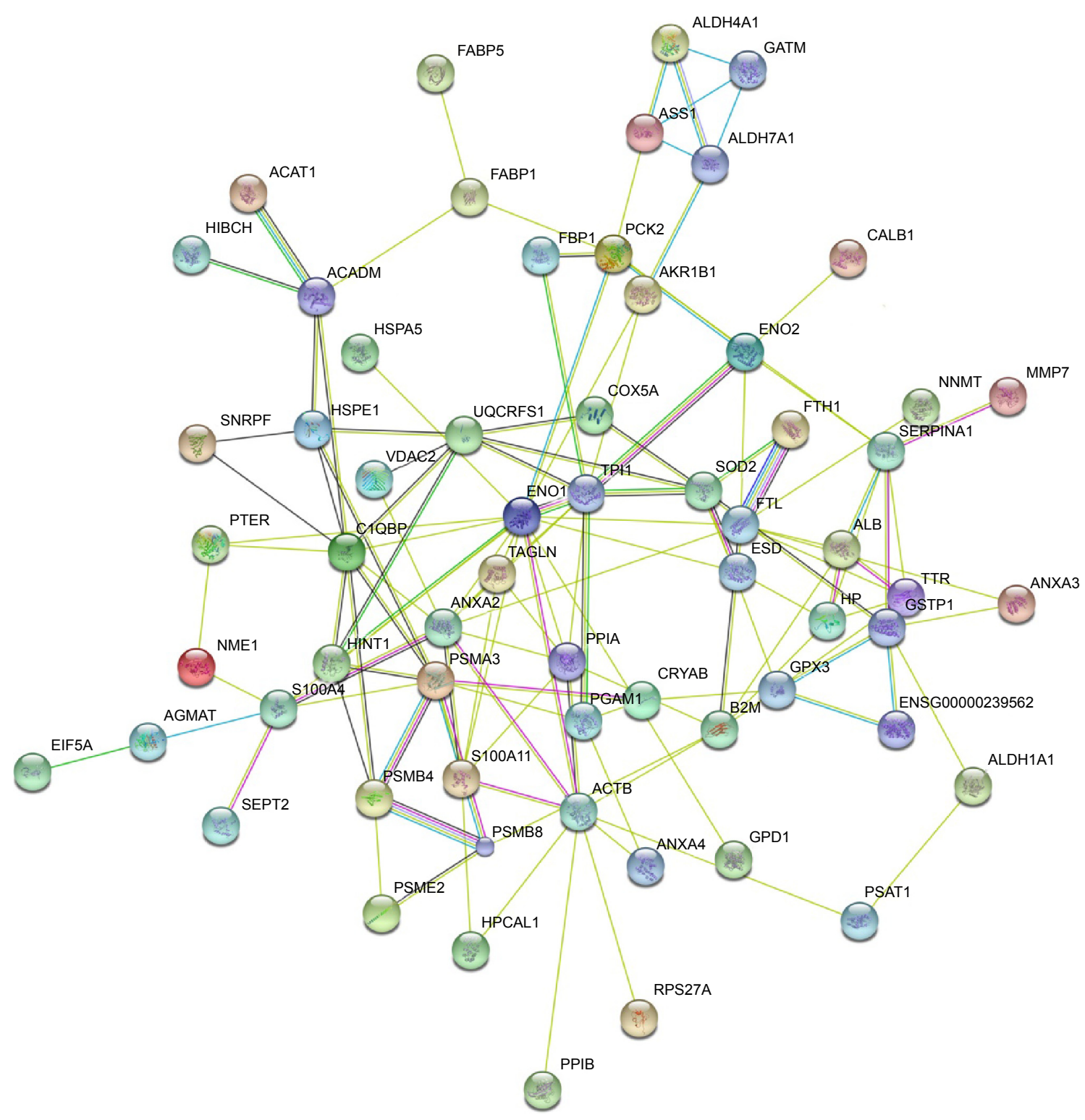

Figure 5 Signaling networks/functional analysis of dysregulated proteins in RCC.

Notes: The identified differentially expressed proteins were analyzed using the STRING tool. ${ }^{14}$ In this map, the network nodes represent proteins. The edges represent predicted functional associations. An edge may be drawn with several different lines. These lines represent the existence of several types of evidence used in predicting the associations. Abbreviation: RCC, renal cell carcinoma.

The finding that glycolysis enzyme levels are most significantly altered in ccRCC is in accordance with the results of other independent studies conducted in different types of cancers. ${ }^{19-21}$ Increased aerobic glycolysis in cancer, a phenomenon known as the Warburg effect, is characterized by increased metabolism of glucose to lactate in the presence of sufficient oxygen. There is a strong connection between this effect and malignant transformation, as evidenced from studies conducted on various tumor cells. ${ }^{22-24}$ p53 and c-myc are considered to be the key tumor genes and the master regulators of metabolism. ${ }^{25,26}$ The pyruvate kinase (PK) gene, which encodes a protein that converts phosphoenolpyruvate to pyruvate with release of an adenosine triphosphate, is the target gene of Myc and HIF-1. ${ }^{27}$ The dimeric form of M2-PK is another protein specific for tumor cells (known as tumor M2-PK), the dimerization seems to be caused by the interaction of M2-PK with certain oncoproteins. It is believed that this adaptive mechanism allows tumor cells to survive in environments where the levels of oxygen and nutrients are not constant. ${ }^{28}$ The interconversion of glycerate3-phosphate and glycerate-2-phosphate is catalyzed by the glycolytic enzyme phosphoglycerate mutase, while enolase catalyzes the conversion of 2-phosphoglycerate to phosphoenolpyruvate. The expression of enolase is regulated both developmentally and specifically within the tissues. Proteome analysis reported in a recent study has shown that 
Table 3 Enriched processes and pathways identified with the kegg database using proteins

\begin{tabular}{|c|c|c|c|}
\hline Pathway & Count & Gene & $P$-value \\
\hline Glycolysis/gluconeogenesis & 9 & ENOI, ENO2, AKRIAI, PGAMI, PGAM4, TPII, PCK2, ALDH7AI, and FBPI & $6.86 \mathrm{E}-16$ \\
\hline Urea cycle and amino metabolism & 4 & GATM, ALDH7A I, ASSI, and AGMAT & $9.96 \mathrm{E}-08$ \\
\hline Propanoate metabolism & 4 & $A L D H 7 A I, A C A D M, H I B C H$, and ACATI & $2.24 \mathrm{E}-07$ \\
\hline Pyruvate metabolism & 4 & $A K R I B I, P C K 2, A L D H 7 A I$, and $A C A T I$ & $4.85 \mathrm{E}-07$ \\
\hline Valine, leucine, and isoleucine degradation & 4 & ALDH7AI, ACADM, HIBCH, and ACATI & 7.77E-07 \\
\hline Proteasome & 4 & PSME2, PSMA3, PSMB4, and PSMB8 & $9.25 \mathrm{E}-07$ \\
\hline PPAR signaling pathway & 4 & FABP5, PCK2, ACADM, and FABPI & $4.25 \mathrm{E}-06$ \\
\hline Beta-alanine metabolism & 3 & $A L D H 7 A I, A C A D M$, and $H I B C H$ & $5.92 \mathrm{E}-06$ \\
\hline Arginine and proline metabolism & 3 & GATM, ALDH4AI, and ASSI & $2.35 \mathrm{E}-05$ \\
\hline Fructose and mannose metabolism & 3 & $A K R I B I, T P I I$, and $F B P I$ & $2.35 \mathrm{E}-05$ \\
\hline Fatty acid metabolism & 3 & $A L D H 7 A I, A C A D M$, and $A C A T I$ & $4.63 \mathrm{E}-05$ \\
\hline Glycerolipid metabolism & 3 & $A K R I B I, A K R I A I$, and $A L D H 7 A I$ & $5.28 \mathrm{E}-05$ \\
\hline Glutathione metabolism & 3 & GSTPI, GSTTI, and GPX3 & $6.36 \mathrm{E}-05$ \\
\hline Antigen processing and presentation & 3 & PSME2, B2M, and HSPA5 & $3.53 \mathrm{E}-04$ \\
\hline Bile acid biosynthesis & 2 & ALDH7AI and SOATI & $9.10 \mathrm{E}-04$ \\
\hline Butanoate metabolism & 2 & $A L D H 7 A I$ and $A C A T I$ & 0.001562 \\
\hline Glycine, serine, and threonine metabolism & 2 & GATM and PSATI & 0.001996 \\
\hline Tryptophan metabolism & 2 & $A L D H 7 A I$ and $A C A T I$ & 0.001996 \\
\hline Metabolism of xenobiotics by cytochrome P450 & 2 & GSTPI and GSTTI & 0.005199 \\
\hline Drug metabolism - cytochrome P450 & 2 & GSTPI and GSTTI & 0.005491 \\
\hline Pyrimidine metabolism & 2 & NME2 and NMEI & 0.009003 \\
\hline Insulin signaling pathway & 2 & $P C K 2$ and $F B P I$ & 0.019038 \\
\hline Oxidative phosphorylation & 2 & UQCRFSI and COX5A & 0.019297 \\
\hline Purine metabolism & 2 & NME2 and NMEI & 0.022799 \\
\hline
\end{tabular}

Note: Enriched processes and pathways identified with the kegg database using proteins which were significantly altered in RCC as compared to normal tissue, with $P<0.05$. Abbreviation: RCC, renal cell carcinoma.

both phosphoglycerate mutase and enolase seem to be differentially overexpressed in human lung squamous carcinoma. Our data suggest that anaerobic glycolysis-related enzyme $\mathrm{PK}$, enolase, is upregulated, whereas the other carbohydrate metabolism-related enzymes, phosphoenolpyruvate carboxy kinase (PCK2) and acetyl-CoA acetyltransferase (ACAT1), are downregulated in $\mathrm{RCC}$, which is consistent with the results from other laboratories. ${ }^{29,30}$ Recently, the role of agents targeting glycolytic activity and glycolysis-linked metabolic processes is being studied for reversal of Warburg effect. ${ }^{31,32}$ Proteasomes and ubiquitin ( $\mathrm{Ub}$ ) are key participants of the energy-dependent, nonlysosomal proteolytic pathway. Previous studies have indicated that cell proliferation and apoptosis are regulated by the $\mathrm{Ub}$-proteasome system. The research community is focusing its efforts on identifying the potential role of certain proteasome inhibitors to act as novel anticancer agents. ${ }^{33}$ In this work, Ubiquitin-40S ribosomal protein $\mathrm{S} 27 \mathrm{a}$ and four members of the proteasome family, PSME2, PSMA3, PSMB4, and PSMB8 were highly expressed in RCC, which is consistent with one previous study. ${ }^{34}$ Other studies have suggested that proteasomes and Ub also have important roles in various nonproteolytic functions. Proteasomes are thought to regulate the translational activities of cytoplasmic mRNAs. ${ }^{35} \mathrm{Ub}$ has been found to have many apparently distinct roles, such as DNA repair, cell cycle progression, modification of polypeptide receptors, and biogenesis of ribosomes. ${ }^{36,37}$

GSH has multiple roles in the body; it is involved in cell differentiation, proliferation, and apoptosis, as well as antioxidant defense and nutrient metabolism. ${ }^{38}$ It has been shown that enzymes involved in GSH metabolism, particularly glutathione $S$-transferase and glutathione peroxidase, play a role in multistage carcinogenesis. ${ }^{39}$ Our results point to significant variations in the GSH-dependent enzyme activity in RCC and support the finding that GSH metabolism is important in RCC onset and progression. Because they have high energy demands, cancer cells are forced to tap into alternative sources of energy, such as fatty acid oxidation and other nonglycolytic pathways. Our findings suggest that the products of fatty acid metabolism have a key role in RCC metabolism. Fatty acid-binding proteins (FABPs) are involved in lipid metabolism, regulation of gene expression, cell signaling, cell growth, and differentiation. ${ }^{40}$ Moreover, FABPs also have an important role in carcinogenesis. ${ }^{41}$ Studies identifying FABP as tumor markers of RCC emphasize the significant role of fatty acid metabolism in the biology of RCC. ${ }^{42,43}$ In comparison with normal tissues, we found that liver-type FABP was expressed at lower rates in 53\% of all tumors, which is consistent with the findings from other studies. ${ }^{44}$ 
The results of this study indicate that other pathways closely associated with gluconeogenesis, such as the urea cycle, pyruvate, pentanoate, and butanoate metabolism, as well as arginine and proline metabolism, are downregulated in ccRCC. In contrast, an increase in one of the key glycolytic enzymes, pyruvate, was observed.

Our study outlines the metabolic phenotype of RCC tissue in detail. Using proteomic analysis to determine which pathways and processes are likely involved in kidney cancer, we found that the glycolysis pathway is significantly altered in ccRCC. Alterations to these pathways will allow clinicians to identify those molecules that affect metabolic regulation, such as activators or inhibitors of HIF-1, mTOR, and AMP kinase, as well as assess the effectiveness of therapy at the molecular level.

\section{Disclosure}

The authors report no conflicts of interest in this work.

\section{References}

1. Chow WH, Dong LM, Devesa SS. Epidemiology and risk factors for kidney cancer. Nat Rev Urol. 2010;7:245-257.

2. Cho E, Adami HO, Lindblad P. Epidemiology of renal cell cancer. Hematol Oncol Clin North Am. 2011;25:651-665.

3. Linehan WM, Walther MM, Zbar B. The genetic basis of cancer of the kidney. J Urol. 2003;170:2163-2172.

4. Veech RL. The therapeutic implications of ketone bodies: the effects of ketone bodies in pathological conditions: ketosis, ketogenic diet, redox states, insulin resistance, and mitochondrial metabolism. Prostaglandins Leukot Essent Fatty Acids. 2004;70:309-319.

5. Greene AE, Todorova MT, Seyfried TN. Perspectives on the metabolic management of epilepsy through dietary reduction of glucose and elevation of ketone bodies. J Neurochem. 2003;86:529-537.

6. Strohman R. Maneuvering in the complex path from genotype to phenotype. Science. 2002;296:701-703.

7. Merida I, Avila-Flores A. Tumor metabolism: new opportunities for cancer therapy. Clin Transl Oncol. 2006;8:711-716.

8. Moreno-Sánchez R, Rodríguez-Enríquez S, Marín-Hernández A, Saavedra E. Energy metabolism in tumor cells. FEBS J. 2007;274: 1393-1418.

9. Locasale JW, Cantley LC. Altered metabolism in cancer. BMC Biol. 2010;8:88.

10. Linehan WM, Srinivasan R, Schmidt LS. The genetic basis of kidney cancer: a metabolic disease. Nat Rev Urol. 2010;7:277-285.

11. Lu ZJ, Liu SY, Yao YQ, et al. The effect of miR-7 on behavior and global protein expression in glioma cell lines. Electrophoresis. 2011;32: 3612-3620.

12. Lu ZJ, Song QF, Jiang SS, et al. Identification of ATP synthase beta subunit (ATPB) on the cell surface as a non-small cell lung cancer (NSCLC) associated antigen. BMC Cancer. 2009;9:16.

13. Kanehisa M. Molecular network analysis of diseases and drugs in KEGG. Methods Mol Biol. 2013;939:263-275.

14. Franceschini A, Szklarczyk D, Frankild S, et al. STRING v9.1: proteinprotein interaction networks, with increased coverage and integration. Nucleic Acids Res. 2013;41:D808-D815.

15. Yuan Y, Xue L, Fan H. Screening of differentially expressed genes related to esophageal squamous cell carcinoma and functional analysis with DNA microarrays. Int J Oncol. 2014;44:1163-1170.

16. Hwa JS, Park HJ, Jung JH, et al. Identification of proteins differentially expressed in the conventional renal cell carcinoma by proteomic analysis. J Korean Med Sci. 2005;20:450-455.
17. Aggelis V, Craven RA, Peng J, et al. Proteomic identification of differentially expressed plasma membrane proteins in renal cell carcinoma by stable isotope labelling of a von Hippel-Lindau transfectant cell line model. Proteomics. 2009;9:2118-2130.

18. Linehan WM, Bratslavsky G, Pinto PA, et al. Molecular diagnosis and therapy of kidney cancer. Annu Rev Med. 2010;61:329-343.

19. Pelicano H, Martin DS, Xu RH, Huang P. Glycolysis inhibition for anticancer treatment. Oncogene. 2006;25:4633-4646.

20. Hammoudi N, Ahmed KB, Garcia-Prieto C, Huang P. Metabolic alterations in cancer cells and therapeutic implications. Chin J Cancer. 2011;30:508-525.

21. Sinreih M, Hevir N, Rizner TL. Altered expression of genes involved in progesterone biosynthesis, metabolism and action in endometrial cancer. Chem Biol Interact. 2013;202:210-217.

22. Chen Z, Lu W, Garcia-Prieto C, Huang P. The Warburg effect and its cancer therapeutic implications. J Bioenerg Biomembr. 2007;39: 267-274.

23. Lopez-Lazaro M. The Warburg effect: why and how do cancer cells activate glycolysis in the presence of oxygen? Anticancer Agents Med Chem. 2008;8:305-312.

24. Bensinger SJ, Christofk HR. New aspects of the Warburg effect in cancer cell biology. Semin Cell Dev Biol. 2012;23:352-361.

25. Wu W, Zhao S. Metabolic changes in cancer: beyond the Warburg effect. Chin J Biochem Biophys. 2013;45:18-26.

26. Guihard S, Ramolu L, Macabre C, et al. The NEDD8 conjugation pathway regulates p53 transcriptional activity and head and neck cancer cell sensitivity to ionizing radiation. Int J Oncol. 2012;41: $1531-1540$

27. Dang CV, Hamaker M, Sun P. Therapeutic targeting of cancer cell metabolism. J Mol Med. 2011;89:205-212.

28. Diaz-Ruiz R, Uribe-Carvajal S, Devin A, Rigoulet M. Tumor cell energy metabolism and its common features with yeast metabolism. Biochim Biophys Acta. 2009;1796:252-265.

29. Perego RA, Bianchi C, Corizzato M, et al. Primary cell cultures arising from normal kidney and renal cell carcinoma retain the proteomic profile of corresponding tissues. J Proteome Res. 2005;4: 1503-1510.

30. Perroud B, Lee J, Valkova N, et al. Pathway analysis of kidney cancer using proteomics and metabolic profiling. Mol Cancer. 2006;5:64.

31. Zhao Y, Liu H, Riker AI, et al. Emerging metabolic targets in cancer therapy. Front Biosci. 2011;16:1844-1860.

32. Zhang Y, Yang JM. Altered energy metabolism in cancer: a unique opportunity for therapeutic intervention. Cancer Biol Ther. 2013;14: 81-89.

33. An J, Sun Y, Fisher M, Rettig MB. Maximal apoptosis of renal cell carcinoma by the proteasome inhibitor bortezomib is nuclear factorkappaB dependent. Mol Cancer Ther. 2004;3:727-736.

34. Kanayama H, Tanaka K, Aki M, et al. Changes in expressions of proteasome and ubiquitin genes in human renal cancer cells. Cancer Res. 1991; 51:6677-6685.

35. Issaenko OA, Bitterman PB, Polunovsky VA, Dahlberg PS. Capdependent mRNA translation and the ubiquitin-proteasome system cooperate to promote ERBB2-dependent esophageal cancer phenotype. Cancer Gene Ther. 2012;19:609-618.

36. Nakamura M, Tanigawa Y. Biochemical analysis of the receptor for ubiquitin-like polypeptide. J Biol Chem. 1999;274:18026-18032.

37. Vaziri SA, Grabowski DR, Hill J, et al. Inhibition of proteasome activity by bortezomib in renal cancer cells is p53 dependent and VHL independent. Anticancer Res. 2009;29:2961-2969.

38. Franco R, Schoneveld OJ, Pappa A, Panayiotidis MI. The central role of glutathione in the pathophysiology of human diseases. Arch Physiol Biochem. 2007;113:234-258.

39. Lusini L, Tripodi SA, Rossi R, et al. Altered glutathione anti-oxidant metabolism during tumor progression in human renal-cell carcinoma. Int J Cancer. 2001;91:55-59.

40. Dutta-Roy AK. Cellular uptake of long-chain fatty acids: role of membrane-associated fatty-acid-binding/transport proteins. Cell $\mathrm{Mol}$ Life Sci. 2000;57:1360-1372. 
41. Ohlsson G, Moreira JM, Gromov P, Sauter G, Celis JE. Loss of expression of the adipocyte-type fatty acid-binding protein (A-FABP) is associated with progression of human urothelial carcinomas. Mol Cell Proteomics. 2005;4:570-581.

42. Teratani T, Domoto T, Kuriki K, et al. Detection of transcript for braintype fatty acid-binding protein in tumor and urine of patients with renal cell carcinoma. Urology. 2007;69:236-240.
43. Forootan FS, Forootan SS, Malki MI, et al. The expression of C-FABP and PPARgamma and their prognostic significance in prostate cancer. Int J Oncol. 2014;44:265-275.

44. Tölle A, Suhail S, Jung M, Stephan C. Fatty acid binding proteins (FABPs) in prostate, bladder and kidney cancer cell lines and the use of IL-FABP as survival predictor in patients with renal cell carcinoma. BMC Cancer. 2011;11:302.

\section{Publish your work in this journal}

OncoTargets and Therapy is an international, peer-reviewed, open access journal focusing on the pathological basis of all cancers, potential targets for therapy and treatment protocols employed to improve the management of cancer patients. The journal also focuses on the impact of management programs and new therapeutic agents and protocols on

\section{Dovepress}

patient perspectives such as quality of life, adherence and satisfaction. The manuscript management system is completely online and includes a very quick and fair peer-review system, which is all easy to use. Visit http://www.dovepress.com/testimonials.php to read real quotes from published authors.

Submit your manuscript here: http://www.dovepress.com/oncotargets-and-therapy-journal 